\title{
More knowledge with the same amount of data: advantage of accounting for parameter correlations in hierarchical meta-analyses
}

\author{
Henni Pulkkinen ${ }^{1,3, *}$, Samu Mäntyniemi ${ }^{1}$, Sakari Kuikkaa ${ }^{1}$, Polina Levontin ${ }^{2}$ \\ ${ }^{1}$ Fisheries and Environmental Management Group, Department of Environmental Sciences, 00014 University of Helsinki, \\ Finland \\ ${ }^{2}$ Imperial College London, South Kensington Campus, London SW7 2AZ, UK \\ ${ }^{3}$ Present address: Finnish Game and Fisheries Research Institute, Rakentajantie 3, 90570 Oulu, Finland
}

\begin{abstract}
In fisheries stock assessment, the amount of information available from a specific stock is often limited, and the resources to gather new data can be insufficient. This is especially the case when management advice is required for by-catch species which are not always well monitored. However, information is often available from other stocks of the same or closely related species. Also, potential correlations between the life history parameters may contain information which is not usually taken into account in stock assessments. Utilizing all available information can be a cost-efficient way to diminish the amount of uncertainty about key parameters for a case with limited data or when constructing an informative prior for a new case study. In hierarchical modeling, different hierarchical levels can be used to connect closely related and more distant stocks. For example, a lower level of hierarchy can link the stocks within the same species, and a higher level, stocks of related species. We use length-weight and length-fecundity datasets from the FishBase database. Using these datasets, we demonstrate how Bayesian hierarchical correlation analysis can improve understanding of fecundity and formalize available knowledge about length-weight and length-fecundity relationships in terms of informative priors for future analysis.
\end{abstract}

KEY WORDS: Bayesian hierarchical models $\cdot$ Correlation $\cdot$ Reducing uncertainty $\cdot$ Informative priors

\section{INTRODUCTION}

Following the principle of precautionary approach, increased uncertainty should lead to more conservative exploitation rates (Fenichel et al. 2008). Conversely, improved information can be expected to lead to higher gains from the exploitation of resources (McDonald \& Smith 1997, Mäntyniemi et al. 2009). Thus, it is desirable to reduce uncertainty about life history parameters in population modeling. The need to give advice for by-catch species provides new challenges for scientists when they interpret available data sets.

Uncertainty can be reduced either by collecting new data or by interpreting existing data more effectively by utilizing biological knowledge to a higher extent. Unfortunately, the resources for data collection for a specific fish stock are often limited or nonexistent. Yet, it might be possible to improve the precision of the estimates by learning from related fish stocks and from species for which larger amounts of data are available. The efficient treatment of the 
vast supplies of publicly available fisheries data is potentially cost-effective, but it also presents a scientific challenge.

Bayesian hierarchical meta-analysis has become popular in many scientific fields, fisheries in particular, because it allows both synthesis and accumulation of knowledge (Myers \& Mertz 1998, Prevost et al. 2003, McCarthy \& Masters 2005). In Bayesian hierarchical meta-analysis, studies are assumed to be (partially) exchangeable implying that there are no known factors that could be used to predict the differences between the studies other than the potential covariates that have been accounted for (Gelman et al. 2004). This perspective allows information from multiple sources to be combined so that we can learn from the past and build on that knowledge (Hilborn \& Liermann 1998). Combining information from different studies within a hierarchical meta-analysis can be more efficient when known correlations among parameters of interest are taken into account instead of treating them as conditionally independent.

Existence of correlations among life history parameters is well known, and there are studies which discuss how this correlation may have arisen from differences in life history strategies (e.g. Rochet 2000, Gislason et al. 2010). To our knowledge, few studies have investigated these correlations with Bayesian methods in order to produce priors for subsequent studies. Helser \& Lai (2004) studied correlations between von Bertalanffy growth parameters and latitude for North American largemouth bass by using Bayesian meta-analytical methods. We have not, however, found any studies that considered correlations between parameters from different life history processes or functions (such as growth and fecundity) with Bayesian methodology.

Relationships between morphology and life history strategies arise as a result of evolutionary processes. Due to physical and environmental constraints, organisms cannot produce large numbers of offspring at an early age. However, species have different life history strategies: some species mature rapidly at smaller body size; others mature slowly at larger body size. Correlated attributes may convey competitive advantages for the different life history strategies found among species (Jennings \& Beverton 1991). Strategies can also vary tremendously within species; for example, in some populations of salmonids, males mature and reproduce either as large adults after years of feeding at sea or as small parrs, which have not migrated from the river and spawn surreptitiously in the middle of conspecifics thousands times their size (Bohlin et al. 1990). The existence of different reproduction strategies, among and within species, indicates that not all combinations of values of life history parameters are equally likely, and life history trade-offs imply that parameters are correlated. He et al. (2006) utilized similar reasoning in their derivation of a prior distribution for the steepness of a stock-recruitment function.

In this paper, we use Bayesian hierarchical models to synthesize information from multiple studies. By considering correlations among parameters we make use of the additional information contained in the biological connections of these parameters. We use this analysis to produce informative distributions for key parameters of an unspecified stock, which could be then used as a prior distribution in subsequent studies. Parameters of length-weight and length-fecundity relationships are used to demonstrate the methodology, with data extracted from the global information system FishBase (Froese \& Pauly 2011).

\section{METHODS}

\section{Example: Correlation between 2 parameters}

To illustrate the potential value of studying these correlations, consider the following graph made with the data taken from FishBase. Fig. 1 shows scatterplot for parameters $\log (a)$ and $b$ in the length-weight relationship of multiple fish species (Froese 2006 discusses and illustrates the relationship between these parameters in greater detail). The different col-

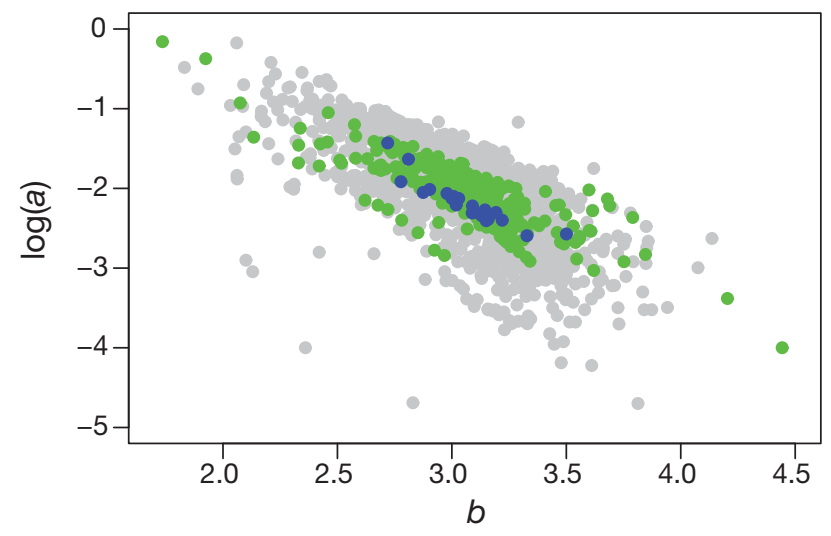

Fig. 1. Scatterplot of length-weight parameters $\log (a)$ and $b$ for multiple fish species. The blue dots represent stocks of Atlantic herring, the green dots represent stocks of different species in the family of Clupeidae, and the grey dots represent stocks of miscellaneous species 
ors in the graph represent different groups of fish species: the blue dots represent stocks of Atlantic herring, the green dots represent stocks of different species in the family of Clupeidae, and the grey dots represent stocks of miscellaneous species.

The parameters $\log (a)$ and $b$ are negatively correlated. We can use the correlation to predict a range of $\log (a)$ values given some information about parameter $b$ for a new fish stock. If the value of $b$ was 3.0, we could say that we believe $\log (a)$ would likely be somewhere on the interval $[-3.3,-1]$. Furthermore, if we knew the new fish stock belongs to the family of Clupeidae, we might consider the possible range of $\log (a)$ values slightly less uncertain, say, $[-2.8,-1.2]$.

\section{General model structure}

Consider $n$ parameters $\theta=\left(\theta_{1}, \ldots, \theta_{n}\right)$ for which there is information about $m$ species and whose correlations are of interest. Let us denote the expected values by $\mu=\left(\mu_{j 1}, \ldots, \mu_{j n}\right)$ as the expected values for the $n$ parameters of species $j$, and $\Sigma$ as a symmetric, positive definite covariance matrix. We assume that $\theta$ follows a multivariate normal distribution (MVN) with expected values $\mu$ and the covariance matrix $\Sigma$ :

$$
\theta \sim \operatorname{MVN}(\mu, \Sigma)
$$

It is computationally convenient to make the choice that $\Sigma^{-1}$ follows the Wishart distribution, since Wishart is the conjugate prior distribution for the inverse of the covariance matrix of multivariate normal distribution (Gelman et al. 2004):

$$
\Sigma^{-1} \sim \text { Wishart }(\Omega, k)
$$

where $\Omega$ is the positive definite scale matrix and $k$ is the degrees of freedom parameter.

For the elements in the matrix of expected values, $\mu$, any suitable prior distributions can be chosen.

\section{Updating knowledge about fecundity}

Next, 2 Bayesian hierarchical models are constructed in which the information about the parameters of length-weight and length-fecundity relationships is analyzed. Both models produce predictive prior distributions for length-fecundity parameters of a new, unspecified stock of round sardinella species. The first model utilises correlations in the analysis and the second does not, which makes it possible to demonstrate the role of the correlations in the inference and to compare the precision of the predictive prior distributions between the models. Thus, the differences in the outputs of these 2 models demonstrate the advantage of taking the correlations into account instead of modeling each parameter separately.

\section{Model with correlation}

Let us formulate the length-weight relationship as:

$$
W=a_{\mathrm{w}} \times L^{b_{\mathrm{W}}}
$$

where $W$ is the weight of a fish, $L$ is the length of a fish, and $a_{\mathrm{w}}$ and $b_{\mathrm{w}}$ are the parameters of this relationship (Gulland 1983). Data from $\mathrm{a}_{\mathrm{w}}-\mathrm{b}_{\mathrm{w}}$ pairs is available in FishBase for almost 3400 species (see Froese \& Pauly 2011).

Similar formulation is given for the length-fecundity relationship as

$$
F=a_{\mathrm{f}} \times L^{b_{\mathrm{f}}}
$$

where $L$ is again the length of a fish, $F$ is the fecundity of a fish, and $a_{\mathrm{f}}$ and $b_{\mathrm{f}}$ are the parameters of this relationship. Data about the length-fecundity parameters in FishBase are quite sparse (Froese \& Pauly 2011), so we hope that learning from the correlations between the length-weight and length-fecundity parameters help to reduce uncertainty about lengthfecundity parameters.

Data from 3 different species are taken into account in the modeling: Atlantic herring Clupea harengus, European pilchard Sardina pilchardus, and round sardinella Sardinella aurita. These species were chosen since they belong to the same family of species (Clupeidae) and because both length-weight and length-fecundity data were available for these species in FishBase (Froese \& Pauly 2011). Data from 69 stocks in total are taken into account in the modeling, from which either length-weight data, lengthfecundity data, or both, are available (Table 1).

Table 1. Clupea harengus, Sardina pilchardus, and Sardinella aurita. Number of stocks of each species for which different type of data are available. $a_{\mathrm{w}}$ and $b_{\mathrm{w}}$ are the parameters of length-weight relationship, $a_{\mathrm{f}}$ and $b_{\mathrm{f}}$ are the parameters of length-fecundity relationship

\begin{tabular}{|lcccc|}
\hline \multirow{2}{*}{ Species } & \multicolumn{5}{c|}{ No. of stocks with available data } \\
& $a_{\mathrm{w}}, b_{\mathrm{w}}$ & $a_{\mathrm{f}}, b_{\mathrm{f}}$ & Both & \\
\hline Clupea harengus & 21 & 7 & 1 & 29 \\
Sardina pilchardus & 21 & 0 & 1 & 22 \\
Sardinella aurita & 17 & 1 & 0 & 18 \\
Total & 59 & 8 & 2 & 69 \\
\hline
\end{tabular}


We assume the conditional joint probability distribution of the 4 parameters of interest follows a multivariate normal distribution:

$$
a_{i j \mathrm{w}}, b_{i j \mathrm{w}}, a_{i j \mathrm{f}}, b_{i j \mathrm{f}} \mid \mu_{j}, \Sigma \sim \operatorname{MVN}\left(\mu_{j}, \Sigma\right)
$$

where vector $\mu_{j}=\left(\mu_{j a_{\mathrm{w}},}, \mu_{j b_{\mathrm{w}},}, \mu_{j a_{\mathrm{f}}}, \mu_{j b_{\mathrm{f}}}\right)$ contains speciesspecific expected values for all 4 parameters, $j$ ranges over species, and $i$ ranges over stocks of each species. Variance-covariance matrix $\Sigma$ contains the covariances $\operatorname{cov}\left(a_{\mathrm{w}}, b_{\mathrm{w}}\right), \ldots, \operatorname{cov}\left(a_{\mathrm{f}}, b_{\mathrm{f}}\right)$, and the variances $\sigma_{a_{\mathrm{W}}}^{2} \sigma_{b_{\mathrm{W}}}^{2} \sigma_{a f}^{2}, \sigma_{b_{\mathrm{f}}}^{2}$ as the diagonal elements. A vague Wishart prior distribution is given for the inverse covariance matrix $\Sigma^{-1}$. We make a simplifying assumption that the variances are the same for all species. This assumption is reasonable since species are related, but the main reason for this arises because of computational convenience. The Wishart distribution is the common choice for the prior of the inverse covariance matrix, but it does not allow specification of unequal variances. Overcoming this problem would require more complicated methods (Barnard et al. 2000).

The expected values $\mu_{j a_{\mathrm{w}}}, \mu_{j b_{\mathrm{W}}}, \mu_{j \mathrm{f}_{\mathrm{f}}}, \mu_{j b_{\mathrm{f}}}$ are considered to be exchangeable among different species $j=$ $1,2,3$. This means the expected values are treated as a random sample from a common distribution defined by mean $\gamma$ and precision $\tau$ parameters:

$$
\begin{aligned}
\mu_{j a_{\mathrm{W}}} & \sim \mathrm{N}\left(\gamma_{a_{\mathrm{w}}}, \tau_{a_{\mathrm{W}}}\right) \\
\mu_{j b_{\mathrm{W}}} & \sim \mathrm{N}\left(\gamma_{b_{\mathrm{W}}}, \tau_{b_{\mathrm{W}}}\right) \\
\mu_{j a f} & \sim \mathrm{N}\left(\gamma_{a f}, \tau_{a \mathrm{f}}\right) \\
\mu_{j b \mathrm{f}} & \sim \mathrm{N}\left(\gamma_{b_{\mathrm{f}}}, \tau_{b \mathrm{f}}\right)
\end{aligned}
$$

Informative hyperprior distributions are given for $\gamma^{\prime}$ s and $\tau$ 's. These distributions have been obtained by examining the available information from FishBase for all the species within the clupeid family, but excluding the information that is used as data in the modeling. Gamma distributions are given for precision parameters $\tau$ to ensure that the values are positive. The model structure and choices for prior distributions can be found in '(A) BUGS code for correlation model' in the supplement at www.intres.com/articles/suppl/m443p029_supp/.

\section{Model without correlation}

In order to evaluate the impact that utilizing correlations has on reducing uncertainty we reproduce the previous model without the correlation structure.

Code for the comparative model is presented in '(B) BUGS code for model without correlation struc- ture' in the supplement. The same prior distributions were given for mean parameters $\gamma$ as in the correlation model. Similarly, the prior distributions for the variances are the same as in the variance-covariance matrix of the correlation model, but the correlations are fixed at zero.

\section{Modeling measurement error}

If information is available on the variances and covariances of the observed point estimates (data), the following procedure could be used for taking into account the measurement error of the parameter estimation.

We define 2 covariance matrices for observed parameters. Covariance matrix

$$
\Sigma_{j \mathrm{w}}=\left[\begin{array}{lc}
\operatorname{var}\left(O_{a_{i j \mathrm{w}}}\right) & \operatorname{cov}\left(O_{a_{i j \mathrm{w}}}, O_{b_{i j \mathrm{w}}}\right) \\
\operatorname{cov}\left(O_{b_{i j \mathrm{w}}}, O_{a_{i j \mathrm{w}}}\right) & \operatorname{var}\left(O_{b_{i j \mathrm{w}}}\right)
\end{array}\right]
$$

contains variances of observed parameters $O_{a i j w}$ and $O_{b_{i j w}}$ for individuals $i=1, \ldots, N_{j}$ of species $j$ as diagonal elements, and the corresponding covariances $\operatorname{cov}\left(O_{a_{i j \mathrm{w}}} O_{b_{i j \mathrm{w}}}\right)=\operatorname{cov}\left(O_{b_{i j \mathrm{w}}{ }^{\prime}} O_{a i j \mathrm{w}}\right)$. Respectively, we can build covariance matrix $\Sigma_{j f}$ for the observed parameters of length-fecundity relationship.

The observed parameter pairs $\left(O_{a_{i j \mathrm{w}}} O_{b_{i j \mathrm{w}}}\right)$ and $\left(O_{a i j f}, O_{b_{i j f}}\right)$ follow 2-dimensional multinormal distributions with the true values $\mathrm{v}_{i j \mathrm{w}}=\left(a_{i j \mathrm{w}}, b_{i j \mathrm{w}}\right)$ and $\mathrm{v}_{i j \mathrm{f}}=$ $\left(a_{i j \mathrm{f}}, b_{i j \mathrm{f}}\right)$ as expected values and with the specified covariance matrices $\Sigma_{j w}$ and $\Sigma_{j f}$ :

$$
\begin{gathered}
O_{a i j \mathrm{w}}, O_{b_{i j \mathrm{w}}} \mid \mathrm{v}_{i j \mathrm{w}}, \Sigma_{j \mathrm{w}} \sim \operatorname{MVN}\left(\mathrm{v}_{i j \mathrm{w}}, \Sigma_{j \mathrm{w}}\right) \\
O_{a i j \mathrm{f}}, O_{b_{i j \mathrm{f}}} \mid \mathrm{v}_{i j \mathrm{f}}, \Sigma_{j \mathrm{f}} \sim \operatorname{MVN}\left(\mathrm{v}_{i j \mathrm{f}}, \Sigma_{j \mathrm{f}}\right)
\end{gathered}
$$

Furthermore, parameters $a_{i j \mathrm{w}}, b_{i j \mathrm{w}}, a_{i j \mathrm{f}}$ and $b_{i j \mathrm{f}}$ follow the multinormal distribution (6).

\section{RESULTS}

Round sardinella is the chosen species for the comparison of the models, since the information for this species is most limited and hence greater gains are anticipated from understanding parameter correlations of the other 2 species. In both model runs (with and without correlations), the posterior predictive distributions of length-fecundity parameters $a_{\mathrm{f}}$ and $b_{\mathrm{f}}$ are estimated for a new, unspecified stock of round sardinella.

The impact of model choice is evident when the joint posterior of parameters $a_{\mathrm{f}}$ and $b_{\mathrm{f}}$ is examined. Strong negative correlation is estimated for these 


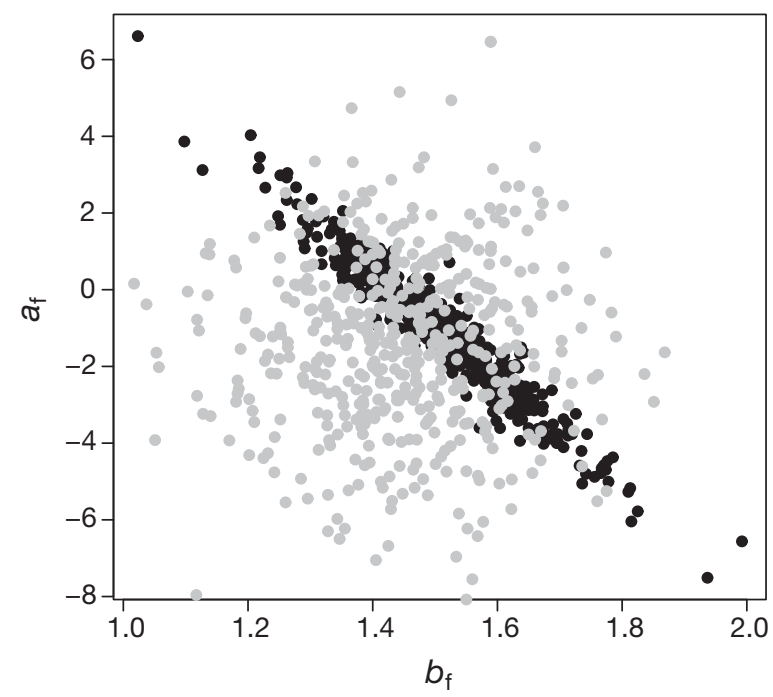

Fig. 2. Sardinella aurita. Scatterplot of a sample of lengthfecundity parameters $a_{\mathrm{f}}$ and $b_{\mathrm{f}}$ for an unspecified round sardinella stock. Black dots represent random draws from the correlation model, and grey dots represent random draws from the model without correlation

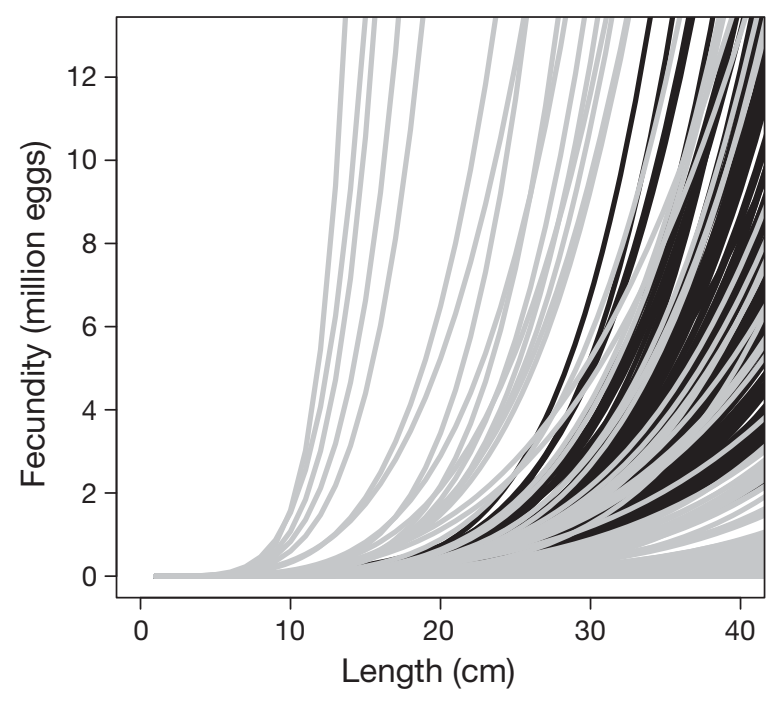

Fig. 3. Sardinella aurita. Samples of randomly drawn lengthfecundity relationship curves for an unspecified round sardinella stock representing the reduction of uncertainty. Black curves represent random draws from the correlation model; grey curves represent random draws from the model without correlation parameters (Fig. 2) in the correlation model, and the predicted distribution of the length-fecundity curve is more precise in this model compared to the model without correlation (Fig. 3). The higher the correlation, the smaller the range of parameter combinations supported by the observations.

The posterior distributions of mean parameters $\mu_{a_{\mathrm{w}}}, \mu_{b_{\mathrm{w}}}, \mu_{\mathrm{af}}$ and $\mu_{b \mathrm{ff}}$ (Fig. 4) show the impact that differently sized datasets have on the uncertainty of the stock specific parameters in the correlation model. The length-weight mean parameters $\mu_{a_{\mathrm{W}}}$ and $\mu_{b_{\mathrm{W}}}$ are estimated with similar precision for all species with both models, whereas the length-fecundity mean parameters $\mu_{a f}$ and $\mu_{\mathrm{bf}}$ are more uncertain for round sardinella than those for Atlantic herring and European pilchard. The central tendencies vary in such a way that the posterior distributions of lengthweight relationship parameters tend to overlap more between species, whereas posteriors of length-fecundity parameters are more distinct.

For each of the European pilchard and round sardinella species, there is only one stock for which lengthfecundity parameters are available.
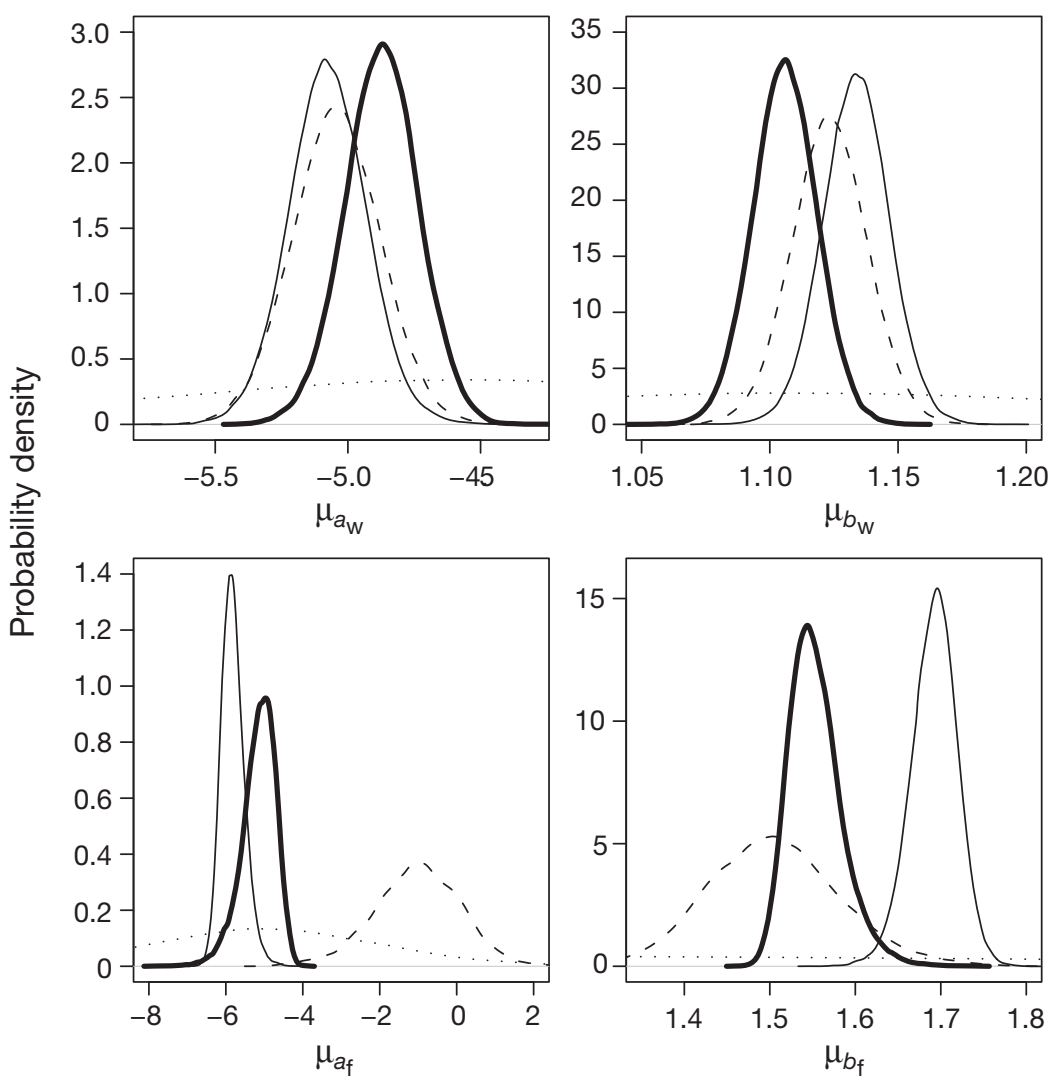

Fig. 4. Clupea harengus, Sardina pilchardus, and Sardinella aurita. Posterior distributions of expected values of length-weight and length-fecundity relationship $a_{\mathrm{w}}, b_{\mathrm{w}}, a_{\mathrm{f}}$ and $b_{\mathrm{f}}$ of different fish species in the correlation model run. Solid bold lines represent Atlantic herring, solid thin lines represent European pilchard, and dashed lines represent round sardinella. Prior distributions are presented with thin dotted line 
For round sardinella, length-weight data is missing for the stock for which length-fecundity parameters are available, which results in more uncertain posterior estimates compared to other species. However, since both the length-weight and length-fecundity data are available for a particular stock of European pilchard, knowledge of species specific correlations is relatively high. For the correlation model, this results in posterior distributions for European pilchard that are nearly as precise as for Atlantic herring, although the available data set is not as large (Table 1). Furthermore, in the model without correlation, the uncertainty of parameters $\mu_{a \mathrm{f}}$ and $\mu_{b_{\mathrm{f}}}$ for European pilchard is much greater (Fig. 5). Thus, only a few observations can give significant information about fecundity when the correlations of stockspecific parameters are taken into account, so a large dataset is not necessarily needed.
Clupea harengus

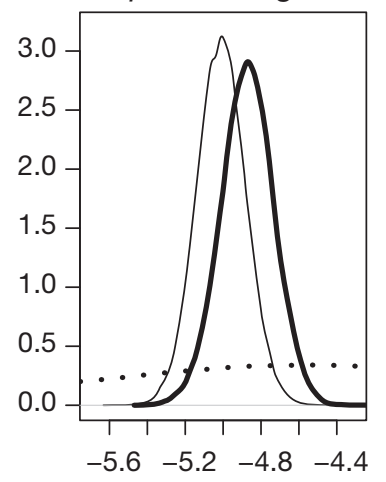

Sardina pilchardus

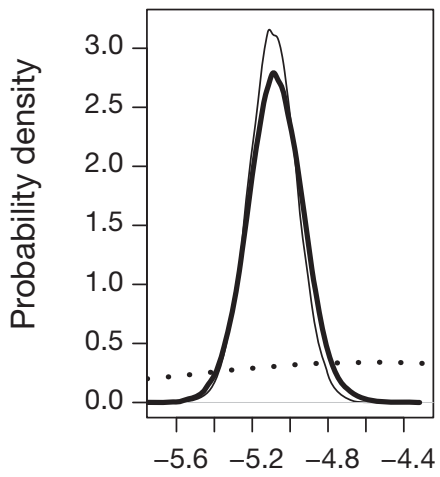

Sardinella aurita

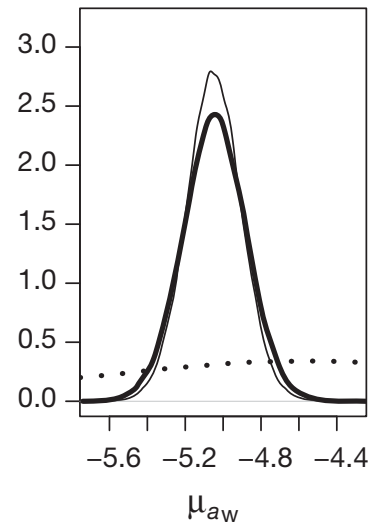

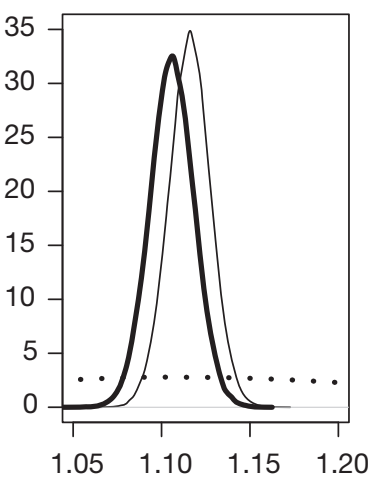
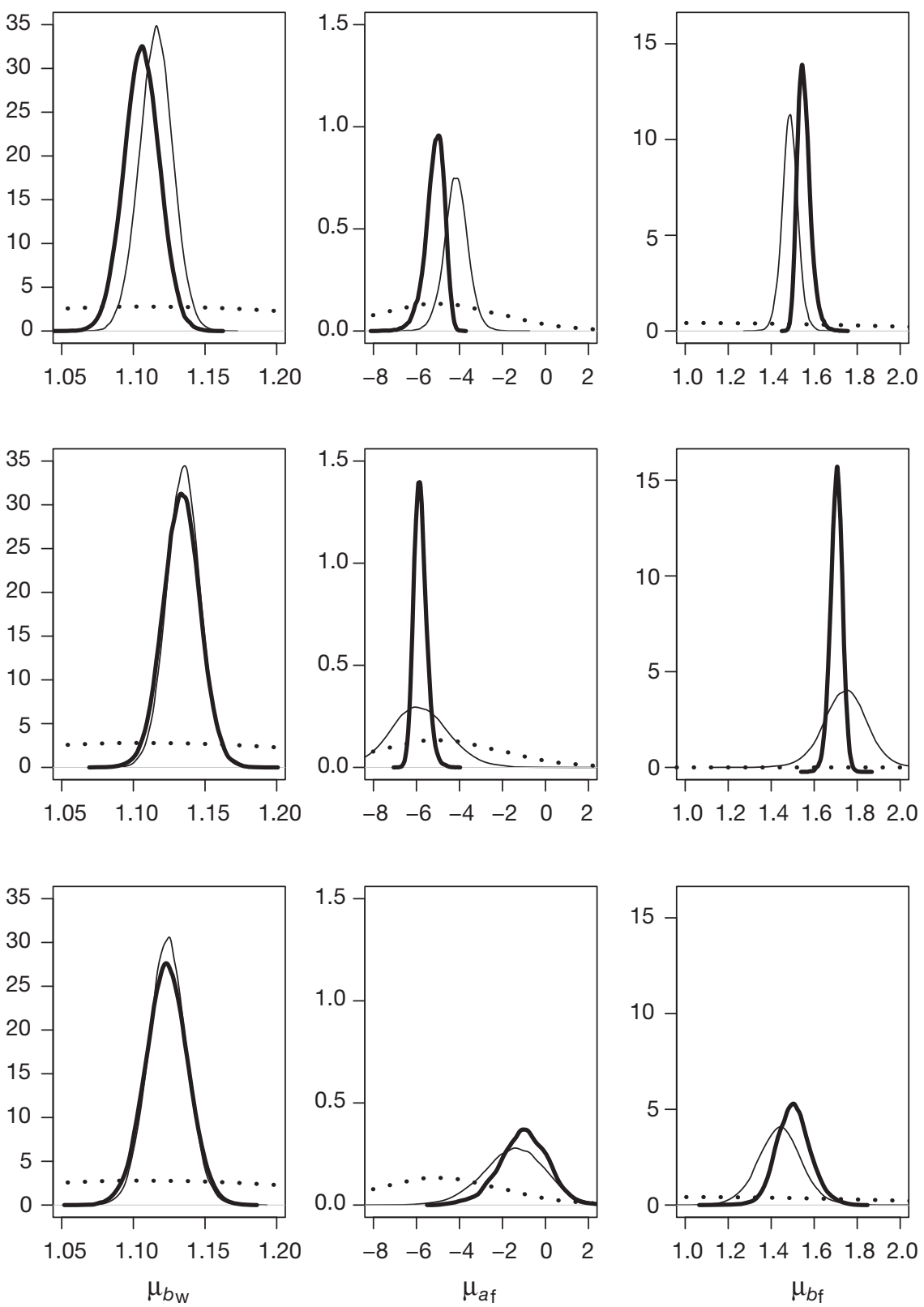

Fig. 5. Clupea harengus, Sardina pilchardus, and Sardinella aurita. Posterior distributions of mean parameters of lengthweight relationship $\mu a_{\mathrm{w}}, \mu b_{\mathrm{w}}$, and length-fecundity relationship $\mu a_{\mathrm{f}}, \mu b_{\mathrm{f}}$ for 3 species and 2 different models. Bold lines represent the posterior distributions of correlation model and thin lines of model without correlation structure. Prior distributions are shown as dotted lines 
Information from length-weight relationship is transmitted to lengthfecundity relationships through the correlation structure on a stock level but it does not lead to more precise knowledge about fecundity on a species level. In case where only information about parameters $a_{\mathrm{w}}$ and $b_{\mathrm{w}}$ are available for a certain species, and nothing is known about parameters $a_{\mathrm{f}}$ and $b_{\mathrm{f}}$, the posterior distributions for $a_{\mathrm{f}}$ and $b_{\mathrm{f}}$ are not much updated from the prior distributions. This situation was studied by adding additional stock into data for which only parameters $a_{\mathrm{w}}$ and $b_{\mathrm{w}}$ were observed and which belong to different species than the ones studied here.

Correlations between the parameters are rather strong, and the influence of the prior distributions is negligible (Fig. 6). The 95\% probability intervals for all 6 correlations is $>0.5$ or smaller than $<-0.5$, indicating a high degree of connection between the variables.

Stock specific mean parameters are presented in Fig. 5, showing the prior distributions and the posterior distributions from both model runs. Posterior distributions for length-weight parameters are rather similar based on both models, although for Atlantic herring the length-fecundity data seems to be strong enough to also update the knowledge about the length-weight parameters. For all 3 species, the difference in results is clearly in favor of the correlation model when inferences are made about length-fecundity parameters. Standard deviation for $\mu_{a f}$ decreases from 0.56 to 0.45 for Atlantic herring, from 1.38 to 0.3 for European pilchard, and from 1.47 to 1.15 for round sardinella.

The posterior distribution of parameter $\mu_{\mathrm{bf}}$ is also more informative when taken from the model using the correlation structure for all 3 species.

\section{Measurement error}

To illustrate the approach of including the measurement error, we simulated data from functions (3) and (4) and estimated the covariance matrices $\Sigma_{\mathrm{w}}$ and $\Sigma_{\mathrm{f}}$ of the observed parameters. We assume that observed parameter pairs $\left(O_{a_{i j \mathrm{w}}} O_{b_{i j \mathrm{w}}}\right)$ and $\left(O_{a i j \mathrm{f}}, O_{b_{i j f}}\right)$ follow the 2-dimensional normal distributions (8) with the same covariance matrices $\Sigma_{\mathrm{w}}$ and $\Sigma_{\mathrm{f}}$ for all the species $j=1,2,3$.

The estimated covariance matrices are

$$
\Sigma_{\mathrm{w}}=\left[\begin{array}{lr}
0.64 & -0.19 \\
-0.19 & 0.06
\end{array}\right] \text { and } \Sigma_{\mathrm{f}}=\left[\begin{array}{ll}
16 & -4.7 \\
-4.7 & 1.44
\end{array}\right]
$$

In this specific case, the differences in the posterior results between model runs with and without measurement error turned out to be minor. The only parameter whose values differ is parameter $a_{f}$ of round sardinella, where the coefficient of variation increased $7 \%$ compared to the model without measurement error. 


\section{DISCUSSION}

We have provided an illustration of how biologically justified model assumptions can effectively decrease the uncertainties about population model parameters. The model introduced herein provides a starting point for future applications that can take into account and utilize potential correlations among life history parameters of fish populations. Because these correlations arise through life history strategies in different species, additional information is likely to be found when any set of fish species is modeled. Also, the model structure can be applied for many available sets of life history parameters.

Not all of the sources of correlations spring from life history evolution. For example, part of the correlation between the life history parameters can arise from the choice of parameters. For example, parameters of length-weight and length-fecundity functions can be expected to have some correlation because length-fecundity relationship can be thought to be composed of a length-weight relationship and a weight-fecundity relationship. Correlation that arises from the fact that both functions include length-weight parameters could be avoided by studying the correlation between length-weight and weight-fecundity curves instead. Other sources of correlations between length-weight and lengthfecundity are due to physics of growth: as a fish grows its organs such as gonads increase proportionally if the shape of the fish is not changing much with age, and bigger volume can accommodate greater number of eggs (Froese 2006). Fecundity is not, however, the only measurement of the reproductive investment: resources can be channeled towards producing larger eggs, rather than a greater number of eggs, and it is known that egg size correlates with better offspring survival (Thorpe et al. 1984). Thus, larger individuals tend to have greater reproductive value even if fecundity does not increase with the size.

If the original length-weight and length-fecundity information were available for all the species, all parameters could be estimated simultaneously in the model with the corresponding covariance matrices. For example, when fitting the regression line between the observed logarithms of weights and lengths, the intercept and slope of the line will be strongly negatively correlated. These types of raw data are unfortunately unlikely to be available for many species, but it would be sufficient if the standard deviations and correlations between estimated parameters were reported in the original publica- tions. This would make it possible to store these values also into databases such as FishBase (Froese \& Pauly 2011), and the information would be available also for the subsequent studies. We have given an example how to treat this kind of information, and although it did not result in any significant difference in this case, we recommend using this approach in case covariance matrices of point estimates are known.

Further, deriving the strength of the correlations among parameters from a small subset of the stocks is not ideal, especially in cases where the biological theory is less supportive of the existence of correlations than in our case. The greater the number of stocks for which information on the correlated parameters is available simultaneously, the stronger the conclusions that can be drawn from the analysis.

When expanding the multivariate normal distribution into highly dimensional spaces, computational problems may become a hindrance. The problems are likely to arise when prior distributions for covariance matrices are specified, as well as during the search for suitable samplers for the estimation of the posterior distributions. Since the Wishart distribution is used as the prior distribution for the inverse of the covariance matrix, utilizing any prior assumptions about the form of standard deviations and the covariances are difficult to implement. There are alternatives for the choice of the prior distribution, as presented in Barnard et al. (2000), which could make the choice of prior easier to justify. Also, the assumption that parameter variances are the same for different species should be critically examined.

In order to utilize historical studies, databases are needed to gather information. In our illustration, FishBase was used as an example of such a database. The advantages of FishBase are that it is easily accessible and can be accessed by anyone through the Internet. Different scientists can contribute to FishBase and obtain greater visibility for their studies, while also adding their share of information to the common pool of knowledge. Finding ways to share information about new studies effectively and efficiently is important so that a wide range of scientists and stakeholders can learn and benefit from them.

The usage of databases brings up many challenges. In the case of FishBase, the length-weight parameters are given as one table for all the species and stocks, but the length-fecundity parameters are presented only in the spawning summary section, separately for each stock, which resulted in a considerable amount of extra work 
when the information was being gathered. Secondly, ensuring that 2 pieces of data are from the same stock can be difficult. This depends on the quality of documentation of a database, which should be as detailed as possible. Quality of the databases should be controlled to ensure reliability of the information as it is used in further scientific studies. Thirdly, although the same biological phenomenon, for example the connection be-tween body length and fecundity, may be examined in 2 different studies, the parameterization of the relationship may differ and so the published parameters may not be comparable. In such situations, the original data should be available in order to produce comparable parameter estimates, which could then be used in the hierarchical modeling. Lastly, databases suffer from publication bias: only those studies containing "statistically significant" results are generally published and so the full range of variations in the parameters of interest are not detected. Statistical significance does not always guarantee biological significance, and vice versa.

By adding more species, more stocks, and different parameters to the model, interesting aspects of the behavior of correlated parameters may be revealed. Studying available data sets more carefully and transferring that knowledge (not only of the parameters from related species but also of correlations between those parameters) might prove advantageous and resource efficient, especially in cases where the amount of available information has been found to be insufficient, and there are inadequate resources to gather more data.

Posterior estimates from hierarchical meta-analysis can be used further as informative prior distributions in various demands of the population modeling. A systematic use of this methodology and available databases offers a way for fisheries science to learn more effectively from all available knowledge. This will benefit the understanding of all species, especially those about which little is known.

Acknowledgements. The research leading to these results received funding from the Finnish Doctoral Programme in Stochastics and Statistics (FDPSS) and the European Union's Seventh Framework Programme (FP7/2007-2013) under grant agreement no. 244706/ECOKNOWS project. However, the paper does not necessarily reflect the views of the European Commission (EC), and in no way anticipates the Commission's future policy in the area. We thank Dr. A. Leach for his valuable comments on the earlier version of this paper.

Editorial responsibility: Konstantinos Stergiou, Thessaloniki, Greece

\section{LITERATURE CITED}

Barnard J, McCulloch R, Meng XL (2000) Modeling covariance matrices in terms of standard deviations and correlations, with application to shrinkage. Statist Sinica 10: 1281-1311

Bohlin T, Dellefors C, Faremo U (1990) Large or small at maturity - theories on the choice of alternative male strategies in anadromous salmonids. Ann Zool Fenn 27: 139-147

Fenichel EP, Tsao JI, Jones ML, Hickling GJ (2008) Real options for precautionary fisheries management. Fish Fish 9:121-137

Froese R (2006) Cube law, condition factor and weightlength relationships: history. Meta-analysis and recommendations. J Appl Ichthyology 22:241-253

Froese R, Pauly D (eds) (2011) FishBase, World Wide Web electronic publication www.fishbase.org (10/2011)

Gelman A, Carling JB, Stern HS, Rubin DB (2004). Bayesian data analysis, 2nd edn. Chapman \& Hall/CRC Press, Boca Raton, FL

Gislason H, Daan N, Rice JC, Pope JG (2010) Size, growth, temperature and the natural mortality of marine fish. Fish Fish 11:149-158

Gulland JA (1983) Fish stock assessment: a manual of basic methods. John Wiley \& Sons, Chichester

He X, Mangel M, MacCall A (2006) A prior for steepness in stock-recruitment relationships, based on an evolutionary persistence principle. Fish Bull 104:428-433

> Helser TE, Lai HL (2004) A Bayesian hierarchical metaanalysis of fish growth: with an example for North American largemouth bass, Micropterus salmoides. Ecol Model 178:399-416

Hilborn R, Liermann M (1998) Standing on the shoulders of giants: learning from experience in fisheries. Rev Fish Biol Fish 8:273-283

Jennings S, Beverton RJH (1991) Intraspecific variation in the life history tactics of Atlantic herring (Clupea harengus L.) stocks. ICES J Mar Sci 48:117-125

> Mäntyniemi S, Kuikka S, Rahikainen M, Kell LT, Kaitala V (2009) The value of information in fisheries management: North Sea herring as an example. ICES J Mar Sci 66: 2278-2283

McCarthy M, Masters P (2005) Profiting from prior information in Bayesian analyses of ecological data. J Appl Ecol 42:1012-1019

McDonald AD, Smith ADM (1997) A tutorial on evaluating expected returns from research for fishery management. Nat Resour Model 10:185-216

> Myers RA, Mertz G (1998) Reducing uncertainty in the biological basis of fisheries management by meta-analysis of data from many population: a synthesis. Fish Res 37:51-60

> Prevost E, Parent E, Crozier W, Davison I and others (2003) Setting biological reference points for Atlantic salmon stocks: transfer of information from data-rich to sparsedata situations by Bayesian hierarchical modelling. ICES J Mar Sci 60:1177-1193

> Rochet MJ (2000) A comparative approach to life-history strategies and tactics among four orders of teleost fish. ICES J Mar Sci 57:228-239

- Thorpe JE, Miles MS, Keay DS (1984) Developmental rate, fecundity and egg size in Atlantic salmon, Salmo salar L. Aquaculture 43:289-305

Submitted: March 11, 2011; Accepted: August 29, 2011

Proofs received from author(s): December 2, 2011 